\title{
Variant Mass for an Accelerated Charged Particle
}

Giovanni Alcocer

Crossref

Independent Researcher, Master in Physics with specialization in Astrophysics and Medical Physics, Professor of Physics, Advanced Mathematics and Science in general.Email: giov_alc_science@hotmail.com

DOI: 10.38177/AJBSR.2020.2304

\section{ABSTRACT}

Albert Einstein wrote in an article entitled: Does a body's inertia depend on its energy content? [1]: "If a body releases energy E in the form of radiation, its mass decreases by $E / c^{2}$. The fact that the energy that leaves from the body is converted into radiation energy makes no difference, so the more general conclusion is reached that the mass of a body is a measure of the content of its energy. It is not impossible that with bodies whose content of energy is highly variable (for example radio salts) the theory can be successfully tested. If the theory corresponds to the fact, radiation conducts inertia between the bodies that emit and absorb it".

Besides, Maxwell's theory shows that electromagnetic waves are radiated whenever charges accelerate [2].

Therefore, the objective of this article is to demonstrate by theory, calculations and results the discovered formula which describe exactly the varian mass of an accelerated charged particle. This charged particle emits electromagnetic radiation which is called the Maxwell Radiation. Also, the article analyzes and establishes about the effects of the variant mass on the particle. In addition, the formula is tested with the electromagnetic radiation emitted for an electron when leaves from the atom. Finally, it is obtained the formula for the power energy emitted for an Accelerated Charged Particle.

Keywords: Maxwell Theory, Einstein Mass-Energy, Variant Mass, Energy, Accelerated Charged Particle, Electromagnetic Radiation, Ionizing Energy Atom, Power Energy.

\section{Introduction}

Firstly, it is presented a research about the mass at the classic limit and at the relativistic case for particles without the emission of electromagnetic radiation. Then, it is demonstrated by theory, calculations and results the discovered formula which describe the mass of an accelerated charged particle emitting electromagnetic radiation. Finally, thought experiments, experimental tests and calculations are presented which confirm the formula theoretically demonstrated.

\section{Theory}

\subsection{Classic Limit}

At the classic limit, the mass of a particle is considered not to change (or changes very little, really this is almost imperceptible). The mass is invariant for particles with velocities much less than the light velocity.

Therefore, $\mathrm{m} \approx \mathrm{m}_{\mathrm{o}}$ during the movement of the particle for velocities much less than the light velocity. Here, $\mathrm{m}_{\mathrm{o}}$ is the mass of the particle at rest.

The second law of Newton is expressed as follows:

$\mathrm{F}=\mathrm{dp} / \mathrm{dt}$

$\mathrm{F}=\mathrm{d}(\mathrm{mv}) / \mathrm{dt}$ where $\mathrm{p}=\mathrm{mv}$ (the formula for momentum)

As $m$ is considered constant, it is obtained:

$\mathrm{F}=\mathrm{mdv} / \mathrm{dt}$ 
$\mathrm{F}=\mathrm{m}_{\mathrm{o}} \mathrm{a}$ where $\mathrm{m}=\mathrm{m}_{\mathrm{o}}$

\subsection{Relativistic Mass}

At the relativistic case, the mass of the particle changes during the movement. It is $m \neq m_{0}$ and the mass is given by the formula of the Relativity Theory:

$m=\frac{m_{o}}{\sqrt{1-\frac{v^{2}}{c^{2}}}}$

Where, $\mathrm{m}_{\mathrm{o}}$ is the mass at rest of the particle.

At this formula, when the velocity $\mathrm{v}$ is increasing, the relativistic mass $\mathrm{m}$ is increasing. If $\mathrm{v}$ approximates to the light velocity $\mathrm{v} \approx \mathrm{c}$, then the value of mass is very high and approaching infinity ( $\infty$ is the representation for so very high values). As conclusion, the velocity $\mathrm{v}$ can never get the light velocity because it implies that the mass increases to the $\infty$. Therefore, we have a limit for the velocity: the light velocity. It is possible to appreciate at the next figure:

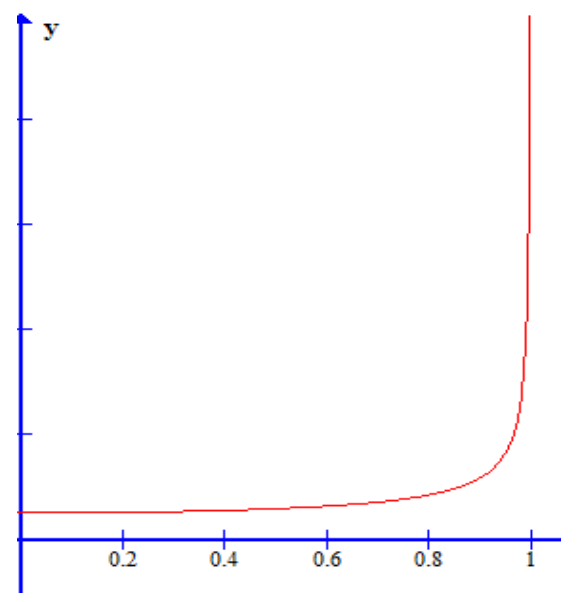

Figure 1: Graph of mass (y axis) versus the rate v/c (x axis) for the relativistic formula

The limit for the velocity is when $\mathrm{v} / \mathrm{c}=1 \mathrm{v} \approx \mathrm{c}$. At the beginning of the graph, $\mathrm{m}$ is approximately constant for values of $\mathrm{v}$ lower than the light velocity $\left(\mathrm{m} \approx \mathrm{m}_{0}=0,511 \mathrm{MeV} / \mathrm{c}^{2}\right)$. After the rate $\mathrm{v} / \mathrm{c}$ is approximately $0.8(\mathrm{v} / \mathrm{c} \approx 0.8)$, the mass starts to increase with a high rate.

It is possible to demonstrate this formula by using energy laws (energy conservation):

The change of the kinetic energy of the particle $\mathrm{dk}$ is transformed in an increasing of the relativistic mass-energy $\mathrm{dE}=\mathrm{c}^{2} \mathrm{dm}$ (by using the famous formula for the energy-mass for every particle: $\mathrm{E}=\mathrm{mc}^{2}$ ). Therefore, it is obtained: $\mathrm{dE}=\mathrm{d} K$

$c^{2} d m=d K$

$d K=d W=F d s=\left(\frac{d p}{d t}\right) d s=d p\left(\frac{d s}{d t}\right)=d(m v) v=v^{2} d m+m v d v$

By replacing this expression in $d K$, it is obtained: 
$c^{2} d m=v^{2} d m+m v d v$

$c^{2} d m=v^{2} d m+m v d v$

$\left(c^{2}-v^{2}\right) d m=m v d v$

$\frac{d m}{m}=\frac{v}{\left(c^{2}-v^{2}\right)} d v$

By doing the respective integration, it is obtained:

$\int_{m_{o}}^{m} \frac{d m}{m}=\int_{0}^{v}-\frac{-2 v}{2\left(c^{2}-v^{2}\right)} d v$

By doing a variable change: $u=c^{2}-v^{2}, d u=-2 v d v$, it is achieved:

$\int_{m_{o}}^{m} \frac{d m}{m}=\int_{c^{2}}^{c^{2}-v^{2}}-\frac{d u}{2 u}$

$\ln (m)-\ln \left(m_{o}\right)=(-1 / 2)\left[\ln \left(c^{2}-v^{2}\right)-\ln \left(c^{2}\right)\right]$

$\ln \left(\mathrm{m} / \mathrm{m}_{\mathrm{o}}\right)=\ln \left[\left(\mathrm{c}^{2}-\mathrm{v}^{2}\right) / \mathrm{c}^{2}\right]^{-1 / 2}$

$\mathrm{m} / \mathrm{m}_{\mathrm{o}}=\left[\left(\mathrm{c}^{2}-\mathrm{v}^{2}\right) / \mathrm{c}^{2}\right]^{-1 / 2}$

$m=\frac{m_{o}}{\sqrt{\left(1-\frac{v^{2}}{c^{2}}\right)}}$

This is the relativistic formula for the relativistic mass of an accelerated particle.

The kinetic formula is obtained as follows:

$c^{2} d m=d K$

And by doing the integration:

$\mathrm{mc}^{2}-\mathrm{m}_{\mathrm{o}} \mathrm{c}^{2}=\mathrm{K}$, then $\quad \mathrm{mc}^{2}=\mathrm{m}_{\mathrm{o}} \mathrm{c}^{2}+\mathrm{K} \quad \mathrm{E}=\mathrm{m}_{\mathrm{o}} \mathrm{c}^{2}+\mathrm{K}$

Which is the formula for the kinetic energy: $\mathrm{K}=\mathrm{mc}^{2}-\mathrm{m}_{\mathrm{o}} \mathrm{c}^{2}$

$K=m_{o} c^{2}\left(\frac{1}{\sqrt{\left(1-\frac{v^{2}}{c^{2}}\right)}}-1\right) \quad K=m_{o} c^{2}\left(\left(1-\frac{v^{2}}{c^{2}}\right)^{-1 / 2}-1\right)$

For very low velocities, it is obtained:

$\left(1-\frac{v^{2}}{c^{2}}\right)^{-1 / 2}=1+\frac{1}{2} \frac{v^{2}}{c^{2}}+\frac{3}{8} \frac{v^{4}}{c^{4}}+$.

$\mathrm{K}=m_{o} c^{2}\left(1+\frac{1}{2} \frac{v^{2}}{c^{2}}+\frac{3}{8} \frac{v^{4}}{c^{4}}+\cdots-1\right)$

$\frac{3}{8} \frac{v^{4}}{c^{4}}$ and other terms are neglected for very low velocities:

$K=\frac{1}{2} m_{o} v^{2}$ (for very low velocities as the classic formula). 


\section{Formula Development: Variant Mass for an Accelerated Charged Particle}

An accelerated charged particle emits electromagnetic radiation and then, the mass decreases [2],[3]. This electromagnetic radiation emitted is the Maxwell radiation for an accelerated charged particle.

It is applied the energy conservation for the development of the formula for the mass of the particle.

The change of the kinetic energy of the particle $\mathrm{dK}$ is transformed in a decreasing of the relativistic mass-energy $\mathrm{dE}=\mathrm{c}^{2} \mathrm{dm}$ (by using the famous formula for the energy-mass for every particle: $\mathrm{E}=\mathrm{mc}^{2}$ ). Thus, it is obtained:

$-c^{2} d m=d K$

$d K=d W=F d s=\left(\frac{d p}{d t}\right) d s=d p\left(\frac{d s}{d t}\right)=d(m v) v=v^{2} d m+m v d v$

$-c^{2} d m=v^{2} d m+m v d v$

$-c^{2} d m=v^{2} d m+m v d v$

$\left(-c^{2}-v^{2}\right) d m=m v d v$

$\frac{d m}{m}=\frac{v}{\left(-c^{2}-v^{2}\right)} d v$

By doing the respective integration, it is obtained:

$\int_{m_{o}}^{m} \frac{d m}{m}=\int_{0}^{v}-\frac{2 v}{2\left(c^{2}+v^{2}\right)} d v$

By doing a variable change: $u=c^{2}+v^{2}, d u=2 v d v$,it is achieved:

$\int_{m_{o}}^{m} \frac{d m}{m}=\int_{c^{2}}^{c^{2}+v^{2}}-\frac{d u}{2 u}$

$\ln (\mathrm{m})-\ln \left(\mathrm{m}_{\mathrm{o}}\right)=(-1 / 2)\left[\ln \left(\mathrm{c}^{2}+\mathrm{v}^{2}\right)-\ln \left(\mathrm{c}^{2}\right)\right]$

$\ln \left(\mathrm{m} / \mathrm{m}_{\mathrm{o}}\right)=\ln \left[\left(\mathrm{c}^{2}+\mathrm{v}^{2}\right) / \mathrm{c}^{2}\right]^{-1 / 2}$

$\mathrm{m} / \mathrm{m}_{\mathrm{o}}=\left[\left(\mathrm{c}^{2}+\mathrm{v}^{2}\right) / \mathrm{c}^{2}\right]^{-1 / 2}$

$m=\frac{m_{o}}{\sqrt{\left(1+\frac{v^{2}}{c^{2}}\right)}}$

which is the formula for the mass of an accelerated charged particle which emits electromagnetic radiation.

The kinetic formula is obtained as follows:

$-c^{2} d m=d K$

and by doing the integration:

$-m c^{2}+m_{0} c^{2}=K$, then $\quad \mathrm{mc}^{2}=\mathrm{m}_{0} \mathrm{c}^{2}-\mathrm{K} \quad \mathrm{E}=\mathrm{m}_{\mathrm{o}} \mathrm{c}^{2}-\mathrm{K}$

which is the formula for the kinetic energy: $\mathrm{K}=\mathrm{m}_{0} \mathrm{c}^{2}-\mathrm{mc}^{2}$ 
$K=m_{o} c^{2}\left(1-\frac{1}{\sqrt{\left(1+\frac{v^{2}}{c^{2}}\right)}}\right) \quad K=m_{o} c^{2}\left(1-\left(1+\frac{v^{2}}{c^{2}}\right)^{-1 / 2}\right)$

For very low velocities, it is obtained:

$\left(1+\frac{v^{2}}{c^{2}}\right)^{-1 / 2}=1-\frac{1}{2} \frac{v^{2}}{c^{2}}+\frac{3}{8} \frac{v^{4}}{c^{4}}+$.

$\mathrm{K}=m_{o} c^{2}\left(1-1+\frac{1}{2} \frac{v^{2}}{c^{2}}-\frac{3}{8} \frac{v^{4}}{c^{4}}+\cdots\right)$

$\frac{3}{8} \frac{v^{4}}{c^{4}}$ and other terms are neglected for very low velocities.

$K=\frac{1}{2} m_{o} v^{2}$ (for very low velocities as the classic formula).

For higher velocities (when $\mathrm{v}$ is approaching to $\infty$ ), the kinetic energy has a maximum value of $\mathrm{m}_{0} \mathrm{c}^{2}$, which correspond to the rest mass of the particle. It is completely emitted as electromagnetic radiation at higher velocities.

$\mathrm{K}=\mathrm{m}_{\mathrm{o}} \mathrm{c}^{2}-\mathrm{mc}^{2}$

$\mathrm{K} \approx \mathrm{m}_{\mathrm{o}} \mathrm{c}^{2} \mathrm{~m} \approx 0$ (when $\mathrm{v} \approx \infty$ ), almost all energy is emitted as electromagnetic radiation.

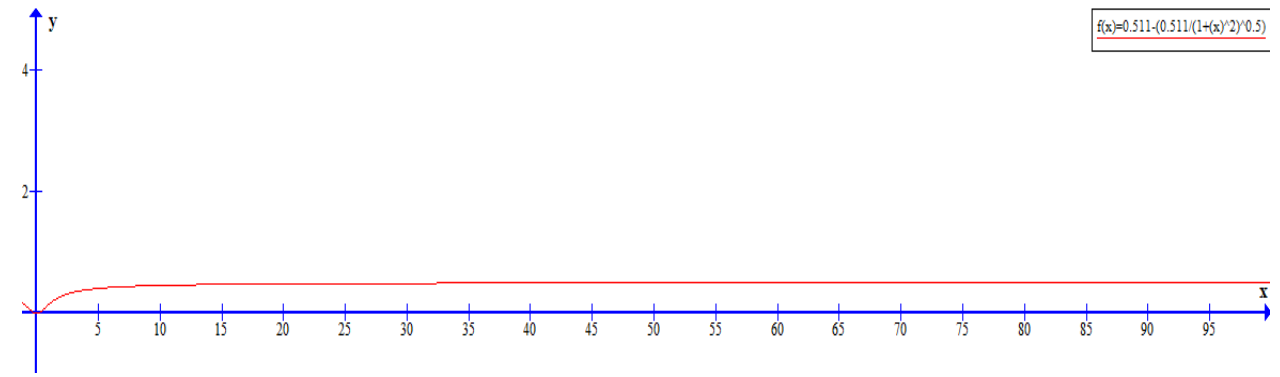

Figure 2: Graph of the kinetic energy (y axis) $(\mathrm{MeV})$ vs the rate v/c (x axis) for an accelerated charged particle

At this formula, there is not limit of velocity for the accelerated charged particle. It is because the particle is losing mass (due to the emission of electromagnetic radiation) during the movement and therefore, it is possible to get each time more velocity without limit. The kinetic energy has a maximum value of $\mathrm{m}_{\mathrm{o}} \mathrm{c}^{2}\left(0,511 \mathrm{MeV} / \mathrm{c}^{2}\right)$ when $\mathrm{v}$ is approaching to $\infty$.

With respect to the mass formula, it is possible to obtain the next conclusion:

$m=\frac{m_{o}}{\sqrt{1+\frac{v^{2}}{c^{2}}}}$

Where, $\mathrm{m}_{\mathrm{o}}$ is the mass at rest of the particle.

At this formula, when the velocity $v$ is increasing, the mass $m$ is decreasing too until $v$ is very high $(\infty)$ and the value of mass is very low (approximately to 0). At this point, almost all the mass is emitted (transformed) as electromagnetic energy. Thus, the velocity $\mathrm{v}$ can get values higher than the light velocity because of the decreasing mass. The lost mass is equivalent to the mass-energy of the electromagnetic radiation emitted. 


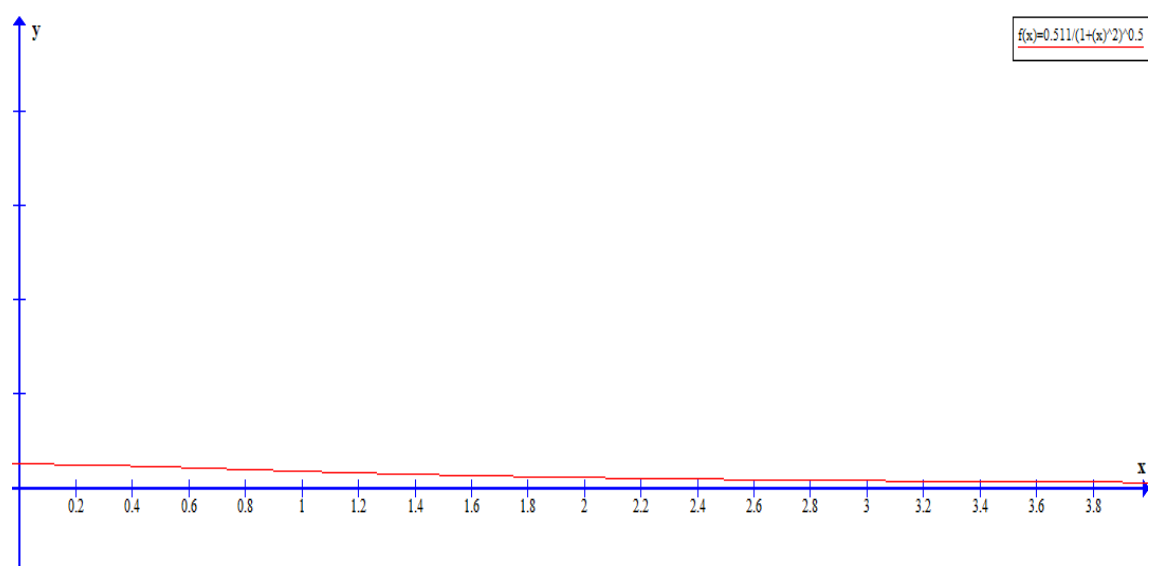

Figure 3: Graph of the mass (y axis) $\left(\mathrm{MeV} / \mathrm{c}^{2}\right.$ ) versus the rate $\mathrm{v} / \mathrm{c}$ (x axis) for an accelerated charged particle. The initial value of the mass is $0,511 \mathrm{MeV} / \mathrm{c}^{2}$

\section{Thought Experiment (Gedanken experiment)}

\subsection{Thought Experiment (Gedanken experiment 1)}

*A rectangular tube of mass $\mathrm{M}$ and length $\mathrm{L}$ is at rest in a system $\mathrm{S}$. A pulse of electromagnetic radiation of energy E is emitted from one extreme of the tube, and then it is absorbed at the other extreme [4]. In this thought experiment, it is demonstrated as follows that the inertia associated with this radiation is $\mathrm{m}=\mathrm{E} / \mathrm{c}^{2}$.

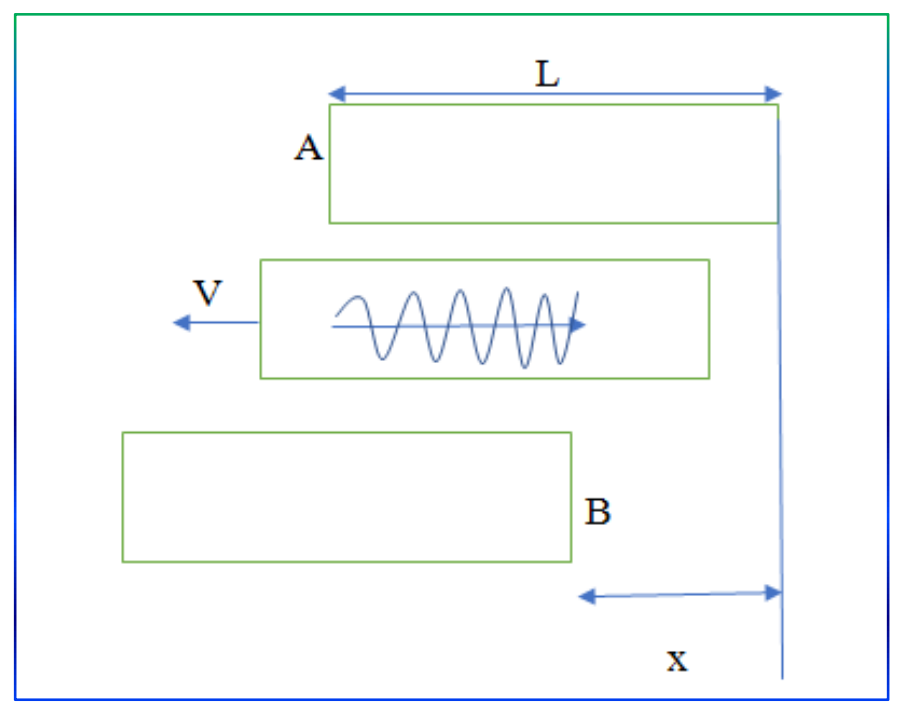

Figure 4: Emission of the electromagnetic radiation pulse (in A) within of the tube of length L. The tube recoils due to the emission of the pulse (momentum conservation). The pulse reaches B and then leaves the tube

Firstly, it is used momentum conservation and then energy conservation as a second form to demonstrate that the inertia associated with this radiation is $\mathrm{m}=\mathrm{E} / \mathrm{c}^{2}$.

\section{Momentum Conservation}

The electromagnetic radiation pulse is emitted to the right. From Maxwell's theory of electromagnetism it is known that an impulse $\mathrm{p}=\mathrm{E} / \mathrm{c}$ is associated with this radiation. In order to preserve the momentum (amount of 
movement), the tube moves back to the left with velocity $\mathrm{v}$. If $\mathrm{m}$ represents the mass associated with the radiated energy, then the mass of the receding tube is $\mathrm{M}-\mathrm{m}$ and the conservation of momentum in $\mathrm{S}$ requires:

$(M-m) v=\frac{E}{c} \quad v=\frac{E}{(M-m) c}$

( $\mathrm{p}=\mathrm{E} / \mathrm{c}$ : impulse of the photon or electromagnetic radiation pulse)

The flight time of the electromagnetic radiation pulse whose velocity is $\mathrm{c}, \mathrm{is} \mathrm{t}=(\mathrm{L}-\mathrm{x}) / \mathrm{c}$ (where $\mathrm{x}$ is the displacement of the tube to the left due to the inertia: recoil of the tube). This time is equal to the recoil time of the tube, $t=x / v$. Combining these times, it is obtained:

$\frac{(L-x)}{c}=\frac{x}{v}$

$\frac{v}{c}=\frac{x}{(L-x)}$

By replacing the value of $v$, it is obtained:

$\frac{E}{(M-m) c^{2}}=\frac{x}{(L-x)}$

Since all forces are internal, the center of mass of the system does not change during the emission and absorption processes. If $m$ is the effective mass emitted by radiation, the center of mass will not change if:

$\mathrm{Mx}=\mathrm{mL}$

Demonstration:

$\mathrm{M}(\mathrm{L})=(\mathrm{M}-\mathrm{m})(\mathrm{L}+\mathrm{x})+\mathrm{mx}$

$\mathrm{ML}=\mathrm{ML}+\mathrm{Mx}-\mathrm{mL}-\mathrm{mx}+\mathrm{mx}$

$\mathrm{Mx}=\mathrm{mL}$

$m=\frac{M x}{L} \quad x=\frac{m L}{M}$

$\frac{E}{(M-m) c^{2}}=\frac{x}{(L-x)}$

By solving for $\mathrm{x}$, it is achieved:

$x=\frac{E L}{(M-m) c^{2}+E}$

And also: $x=\frac{m L}{M}$

By equating both equations and solving for $m$, it is obtained:

$\frac{E L}{(M-m) c^{2}+E}=\frac{m L}{M}$

$m^{2}-\left(\frac{E}{c^{2}}+M\right) m+\frac{M E}{c^{2}}=0$ 
There are two mathematical solutions:

$\mathrm{m}=\mathrm{E} / \mathrm{c}^{2}$ and $\mathrm{m}=\mathrm{M}$, but the second solution is not valid because $\mathrm{m} \neq \mathrm{M}$.

Thus, it has been shown that the electromagnetic radiation pulse has an associated mass $\mathrm{m}=\mathrm{E} / \mathrm{c}^{2}$ (which previously in the case of the tube belonged to the tube (it is the lost mass in the tube) and was emitted as electromagnetic radiation).

It is possible to get a very important conclusion [4]:

How does the message of moving to the left arrives to the right extreme of the tube after the left extreme of the tube goes back?

This message of moving to the left for the right extreme of the tube must travel much faster than the light velocity to win the pulse emitted. If the tube were to move like a rigid body the message would travel at infinite velocity. The entire tube does not go back rigidly.

So, this appears that the message of mass lost in the tube $m$ (mass-energy emitted as a pulse of electromagnetic energy travelling at the light velocity) is transmitted along the tube at infinite velocity. This is in accordance with the mass formula for a charged particle that emits electromagnetic radiation because the particle loses approximately all its mass at very high speeds and when $\mathrm{v}$ approaches infinity the mass approaches zero. In other words, this lost mass in the tube is related to the infinite transmission of the message along the tube (the lost mass is transmitted along the tube at infinite velocity).

\section{Energy Conservation}

$\mathrm{mc}^{2}=\mathrm{pc} \quad$ The energy of the photon emitted is $\mathrm{E}=\mathrm{pc}$ and $\mathrm{m}$ is the lost mass of the tube and emitted as electromagnetic radiation.

$\mathrm{M}-\mathrm{m}$ is the mass of the tube after the emission of the electromagnetic pulse.

$\mathrm{E}=\mathrm{hf} \quad$ (energy of the photon)

$\mathrm{E}=\mathrm{hc} / \lambda(\mathrm{c}=\lambda \mathrm{f}$ for the light velocity $)$

$\mathrm{p}=\mathrm{h} / \lambda($ for a photon)

Therefore, E=pc for a photon.

Also, it is possible to obtain the same from the Dirac equation:

$E^{2}=m^{2} c^{4}+p^{2} c^{2}$

As the mass of the photon is $\mathrm{m}=0$, then, it is obtained $\mathrm{E}=\mathrm{pc}$

Thus, the energy of the lost mass of the tube is equal to the energy of the photon emitted:

$\mathrm{mc}^{2}=\mathrm{pc}$

Therefore: $\mathrm{p}=\mathrm{mc}$ and because the energy of the photon is $\mathrm{E}=\mathrm{pc}$, it is obtained: 
$\mathrm{E}=\mathrm{mc}^{2}$ and the associated mass of the photon emitted is $\mathrm{m}=\mathrm{E} / \mathrm{c}^{2}$, where $\mathrm{E}$ is the energy of the photon and $\mathrm{m}$ is the lost mass on the tube and also the associated mass-energy of the photon emitted.

\subsection{Thought Experiment (Gedanken experiment 2)}

*Consider a body B at rest in system S. Two pulses of radiation, each one with E/2 energy, are emitted from B, one pulse moving in the positive $\mathrm{x}$ direction and the other in the negative $\mathrm{x}$ direction. These pulses are emitted by $\mathrm{B}$, whose energy then decreases by a quantity $\mathrm{E}$ and $\mathrm{E} / 2$ is the energy of each pulse emitted. For reasons of symmetry, B must remain at rest in S. Now, let us consider the same process with respect to $S^{\prime}$, which moves with a constant velocity $\mathrm{v}$ with respect to $\mathrm{S}$ in the negative direction of $\mathrm{y}$. In this case, $\mathrm{B}$ moves with velocity $\mathrm{v}$ in the positive direction of $y^{\prime}$. The radiation pulses are partly directed upward, forming an angle $\alpha$ with the $x^{\prime}$ axis. The velocity of B remains constant in $\mathrm{S}^{\prime}$ (after the emission of the radiation) since B remains at rest in $\mathrm{S}$ during this process.

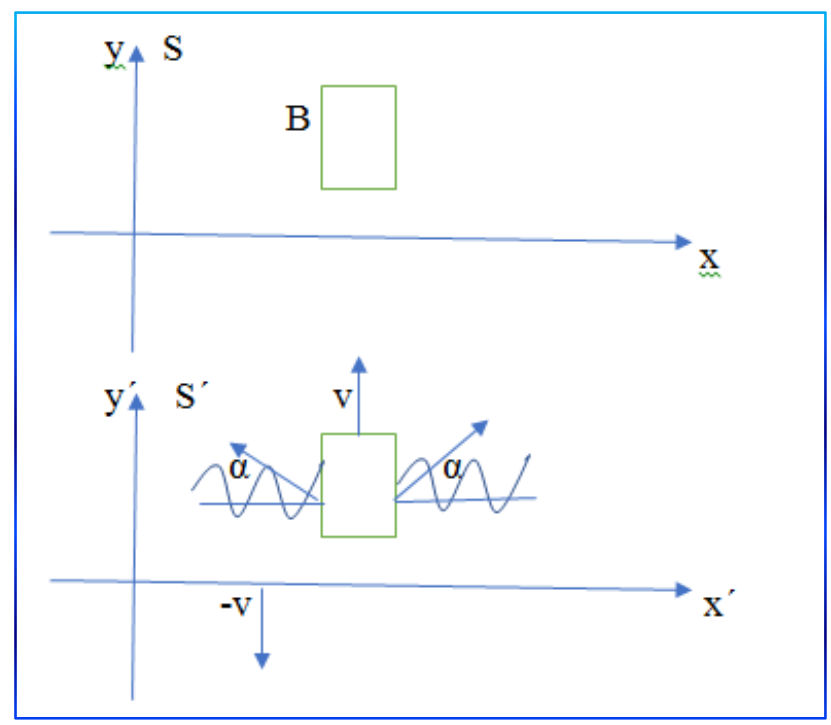

Figure 5: The body B is at rest in $\mathrm{S}$. Then two pulses of radiation are emitted in the directions shown by the arrows. In $\mathrm{S}^{\prime}$ that moves with respect to $\mathrm{S}$ with velocity $\mathrm{v}$ in the negative direction of $\mathrm{y}$, the body $\mathrm{B}$ moves with velocity $\mathrm{v}$ along $+\mathrm{y}^{\prime}$ and the pulses are directed making an angle $\alpha$ with the $\mathrm{x}$-axis

Now, let's apply the law of momentum conservation in the process in $S^{\prime}$. The momentum of $B$ in $S^{\prime}$ before the emission is $\mathrm{Mv}$ (from classic mechanics) along the positive direction of $\mathrm{y}^{\prime}$. In other words, before the emission takes place, the $\mathrm{y}^{\prime}$ component of the impulse of $\mathrm{B}$ is: $\mathrm{Mv}$

After the emission, the body $\mathrm{B}$ has mass $\mathrm{M}^{\prime}$ and the momentum is $\mathrm{M}^{\prime} \mathrm{v}$ along the positive direction $\mathrm{y}^{\prime}$. Each radiation pulse emitted has an energy of $E / 2$ and a momentum $p=E / 2 c(p=E / c$ from classic electromagnetism) with the components of momentum being equal to $(\mathrm{E} / 2 \mathrm{c}) \sin \alpha$.

Therefore, by applying momentum conservation after the emission, it is obtained:

$\mathrm{M}^{\prime} \mathrm{v}+2(\mathrm{E} / 2 \mathrm{c}) \sin \alpha$

By equating the components $\mathrm{y}^{\prime}$ of the impulse before the absorption with those of after absorption, it is achieved:

$M v=M^{\prime} v+2(E / 2 c) \sin \alpha$ 
$\sin \alpha \approx \alpha=\mathrm{v} / \mathrm{c}(\mathrm{v}<<\mathrm{c})$ (classic absorption)

$\mathrm{Mv}=\mathrm{M}^{\prime} \mathrm{v}+2(\mathrm{E} / 2 \mathrm{c})(\mathrm{v} / \mathrm{c})$

$\mathrm{M}=\mathrm{M}^{\prime}+\left(\mathrm{E} / \mathrm{c}^{2}\right)$

$\mathrm{M}-\mathrm{M}^{\prime}=\mathrm{E} / \mathrm{c}^{2}$

Therefore, the decrease in mass $\Delta \mathrm{M}=\mathrm{M}-\mathrm{M}^{\prime}$ of the body $\mathrm{B}$ is linked to the energy $\mathrm{E}$ of the two photons emitted (summed energy) (this energy is also the lost energy of the body B). Therefore:

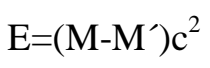

$\mathrm{E}=\Delta \mathrm{Mc}^{2}$

$\Delta \mathrm{M}=\mathrm{E} / \mathrm{c}^{2}$ and the associated mass of the photon emitted is $\Delta \mathrm{M}=\mathrm{E} / \mathrm{c}^{2}$, where $\mathrm{E}$ is the energy of the photon emitted and $\Delta \mathrm{M}$ is also the lost mass on the body $\mathrm{B}$.

\section{Experimental Test}

Every experiment where it is possible to measure the kinetic energy for an accelerated charged particle emitting electromagnetic radiation can be a test for the formula:

$K=m_{o} c^{2}\left(1-\frac{1}{\sqrt{\left(1+\frac{v^{2}}{c^{2}}\right)}}\right)$

In fact, a signal or prove of this formula is the maximum kinetic energy of the accelerated charged particle that can be measured. It is $m_{o} c^{2}$ if there is not additional energy given to the particle at the beginning.

This energy can be measured at the PMT (photomultiplier) as the maximum kinetic energy for an electron for example.

Also, it is intrinsic characteristics of the charged particle and independent of the experimental configuration or material used as detector at the experiment for example.

This maximum kinetic energy for the electron appears in the experiment of muons and neutral kaons from ultra dense hydrogen by lepton pair production [5].

At this experiment, we have the next decays:

$\mu \pm \rightarrow \mathrm{N}\left(\mathrm{e}^{+}+\mathrm{e}^{-}\right)$

$\mu \pm \rightarrow \mu \pm+\mathrm{N}\left(\mathrm{e}^{+}+\mathrm{e}^{-}\right)+\mathrm{KE} \quad \mathrm{KE}: 10-100 \mathrm{MeV}$ dissipation

$\mu \pm \rightarrow \mathrm{e} \pm+$ neutrinos $+\mathrm{N}\left(\mathrm{e}^{+}+\mathrm{e}^{-}\right)+\mathrm{KE} \quad \mathrm{KE}<105 \mathrm{MeV}$ dissipation

The energy for the pair-production is derived from the kinetic energy of the muons of $10-100 \mathrm{MeV}$ and from the decay of the muons at $105 \mathrm{MeV}$. As the dissipation energy is around $100 \mathrm{MeV}$ and each pair production process dissipates around $2.04 \mathrm{eV}$, then we have around 50 lepton pair per muon [5]. The kinetic energy given to the electron-positron pairs increases until that energy is equal to $2 \times 511 \mathrm{keV}$ when a new pair can be produced and 
energy above this limit gives a new lepton pair [5]. Therefore, at the beginning the electron has an energy equals to the rest mass of the particle. The accelerated electron has many interactions with the atoms and nuclei of the material used as detector but nevertheless the maximum kinetic energy measured at the PMT is approximately 511 $\mathrm{keV}$ [5]. It is the radiated energy for the electron during the path. Also, the same electron energy distribution is found for all materials used as converters [5]. Thus, it depends of an electron property and no of the converter material used [5]. It is in accordance with the kinetic formula for the electron which we have demonstrated before.

It is a intrinsic property of the electron: the way that the electron radiates the energy, specifically the electrodynamic mass. Just the high energy cut-off is close to the rest mass of the electron $511 \mathrm{keV}$, which is a confirmation of the nature of electron when it shares the electromagnetic radiation. Therefore, the kinetic energy of leptons entering and measured at the PMT is approximately $511 \mathrm{keV}$ (zero-signal cut-off above $511 \mathrm{keV}$ [5]) which is in agreement with the formula demonstrated.

\section{Hydrogen atom: Emission of Electromagnetic Radiation for the Electron at the Atom}

In order to test the mass formula for an accelerated charged electron for the Hydrogen atom, some calculation by using Quantum Mechanics are done. After, the mass results for both methods are compared.

The formulas for velocity, radius and energy for an electron at the quantized atom [6] are as follows:

$v=\frac{Z e^{2}}{2 \varepsilon_{o} n h}$

$r=\frac{n^{2} h^{2} \varepsilon_{o}}{\pi m_{o} Z e^{2}}$

$E=\frac{-z^{2} e^{4} m_{o}}{8 \varepsilon_{o}^{2} h^{2} n^{2}}$

$\mathrm{Z}$ : atomic number of the atom

e: charge of the electron

$\varepsilon_{0}:$ vacuum permittivity

$\mathrm{n}$ : main quantum number, electron energy level, orbit of the electron

h: Planck constant

$\mathrm{m}_{\mathrm{o}}$ : rest mass of the electron

Values of constants:

$\mathrm{Z}=1$

$\mathrm{h}=6,63 * 10^{-34} \mathrm{~J}-\mathrm{s}$

$\varepsilon_{0}=8,85^{*} 10^{-12} \mathrm{Farad} / \mathrm{m}$

$\pi=3,1416$

$\mathrm{m}_{\mathrm{o}}=9,11 * 10^{-31} \mathrm{~kg}$ 
$e=1,6 * 10^{-19} \mathrm{C}$

$\mathrm{r}_{\mathrm{o}}=0,528 \dot{A}$ Bohr radius, radius for the first level of the Hydrogen atom

$1 \dot{A}=10^{-10} \mathrm{~m}$

\begin{tabular}{|l|r|}
\hline $\mathrm{h}$ & $6,63 * 10^{-34} \mathrm{~J}-\mathrm{s}$ \\
\hline$\varepsilon_{\mathrm{o}}$ & $8,85^{*} 10^{-12} \mathrm{Farad} / \mathrm{m}$ \\
\hline$\pi$ & 3,1416 \\
\hline $\mathrm{m}_{\mathrm{o}}$ & $9,11 * 10-31 \mathrm{~kg}$ \\
\hline $\mathrm{e}$ & $1,6^{*} 10^{-19} \mathrm{C}$ \\
\hline $\mathrm{r}_{\mathrm{o}}$ & $0,528 \quad \dot{A}$ \\
\hline
\end{tabular}

Table 1: Table of physical constants

First energy level Hydrogen atom: $-13,6 \mathrm{eV} \quad \mathrm{n}=1$

Second energy level Hydrogen atom: $\quad-3,4 \mathrm{eV} \quad \mathrm{n}=2$

Therefore, if the electron jumps from the first level to the second level, it must gain an energy of 10,2 eV (energy difference of the two levels).

If the electron jumps from the second level to the first level, it must lose and energy of $10,2 \mathrm{eV}$.

If the electron jumps from the second level to the first level, the mass of the electron must lose this equivalent mass-energy. And the lost mass of the electron (which is equivalent to the mass-energy of the electromagnetic radiation emitted) occurs during the transition from the second level to the first level converted as kinetic energy.

In mathematical formulation, it is as follows:

$\left(\mathrm{m}_{\mathrm{o}}-\mathrm{m}\right) \mathrm{c}^{2}=\mathrm{hf}=\mathrm{K}$

$\mathrm{E}=\mathrm{hf}$ : energy of the photon emitted (electromagnetic radiation).

It is in coincidence with the formula for the kinetic energy for an Accelerated Charged Particle.

Thus, we are going to proceed to test the mass formula:

If the electron at the first level $(n=1)$ leaves from the atom, then the ionizing emery is equal to $-13,6 \mathrm{eV}$. It corresponds to the energy emission of the electron. Therefore, the mass of the electron after losing this mass-energy emission is:

$m c^{2}=m_{o} c^{2}-h f$

$\mathrm{mc}^{2}=511875-13,6$

$=511861,4 \mathrm{eV}$

For other hand, the mass electron calculation with the mass formula for an accelerated charged particle is as follows: 
$m=\frac{m_{o}}{\sqrt{1+\frac{v^{2}}{c^{2}}}}$

The velocity is given by this formula:

$v=\frac{Z e^{2}}{2 \varepsilon_{o} n h}$

It is interesting to mention that this formula doesn't include the mass of the particle. So, the orbits have specific values for the particle independent of the mass of it.

By replacing the values for $\mathrm{Z}(\mathrm{Z}=1), \mathrm{e}, \varepsilon_{\mathrm{o}}, \mathrm{n}(\mathrm{n}=1)$, h, it is obtained:

$\mathrm{v}=2181489,72 \mathrm{~m} / \mathrm{s}$

By replacing this value at the mass formula, it is achieved:

$m c^{2}=\frac{m_{o} c^{2}}{\sqrt{1+\frac{v^{2}}{c^{2}}}}$

$\mathrm{mc}^{2}=511861,4 \mathrm{eV}$ It is the same value that the last calculation by using quantum mechanics.

It is possible to do the same for the second level of the Hydrogen atom.

The ionizing energy for the electron at the second level is: $-3,4 \mathrm{eV}$.

If the electron at the second level $(n=2)$ leaves from the atom, the ionizing energy is equal to $-3,4 \mathrm{eV}$. It corresponds to the energy emission of the electron.

Therefore, the mass of the electron after losing this mass-energy emission is:

$\mathrm{mc}^{2}=\mathrm{m}_{\mathrm{o}} \mathrm{c}^{2}-\mathrm{hf}$

$\mathrm{mc}^{2}=511875-3,4$

$=511871,6 \mathrm{eV}$

For other hand, the mass electron calculation with the mass formula for an accelerated charged particle is as follows:

$m=\frac{m_{o}}{\sqrt{1+\frac{v^{2}}{c^{2}}}}$

The velocity is given by this formula:

$v=\frac{Z e^{2}}{2 \varepsilon_{o} n h}$

By replacing the values for $\mathrm{Z}(\mathrm{Z}=1), \mathrm{e}, \varepsilon_{\mathrm{o}}, \mathrm{n}(\mathrm{n}=2), \mathrm{h}$, it is obtained:

$\mathrm{v}=1090744,859 \mathrm{~m} / \mathrm{s}$

By replacing this value at the mass formula, it is achieved: 
$m c^{2}=\frac{m_{o} c^{2}}{\sqrt{1+\frac{v^{2}}{c^{2}}}}$

$\mathrm{mc}^{2}=511871,6 \mathrm{eV}$ It is the same value that the last calculation by using quantum mechanics. It is showed at the next table the values of the velocities, radius, energy of the ionization for the different levels of energy of the hydrogen atom. Also, it is showed the mass of the electron after the emission of the electromagnetic radiation by using quantum mechanics $\left(\mathrm{mc}^{2}=\mathrm{m}_{0} \mathrm{c}^{2}-\mathrm{hf}\right)$ and for the formula of the variant mass for an accelerated charged particle: $m c^{2}=\frac{m_{o} c^{2}}{\sqrt{1+\frac{v^{2}}{c^{2}}}}$. It is possible to confirm the accuracy of the formula demonstrated theoretically. Besides, the table showed that when the velocity decreases (at the different levels of energy of the Hydrogen atom) the mass increases. Also, levels which are closest to the nucleus have higher velocities than the farthest.

\begin{tabular}{|r|c|c|c|r|r|r|}
\hline $\mathrm{n}$ & & $\mathrm{v}$ & $\mathrm{r}$ & $\mathrm{E}=\mathrm{hf}$ (ionizatin energy) & $\mathrm{mc}^{2}=\mathrm{m}_{0} \mathrm{c}^{2}-\mathrm{hf}$ & $m c^{2}=\frac{m_{o} c^{2}}{\sqrt{1+\frac{v^{2}}{c^{2}}}}$ \\
\hline 1 & 2181489,719 & $5,30961 \mathrm{E}-11$ & $-13,54798602$ & 511861,452 & 511861,4674 \\
\hline 2 & 1090744,859 & $2,12384 \mathrm{E}-10$ & $-3,386996504$ & 511871,613 & 511871,6168 \\
\hline 3 & 727163,2396 & $4,77865 \mathrm{E}-10$ & $-1,50533178$ & 511873,4947 & 511873,4963 \\
\hline 4 & 545372,4297 & $8,49537 \mathrm{E}-10$ & $-0,846749126$ & 511874,1533 & 511874,1542 \\
\hline 5 & 436297,9438 & $1,3274 \mathrm{E}-09$ & $-0,541919441$ & 511874,4581 & 511874,4587 \\
\hline 6 & 363581,6198 & $1,91146 \mathrm{E}-09$ & $-0,376332945$ & 511874,6237 & 511874,6241 \\
\hline 7 & 311641,3884 & $2,60171 \mathrm{E}-09$ & $-0,276489511$ & 511874,7235 & 511874,7238 \\
\hline
\end{tabular}

Table 2: Values of the velocities, radius, energy of the ionization for the different levels of energy of the hydrogen atom. Also, it is showed the mass of the electron after the emission of the electromagnetic radiation by using quantum mechanics and for the formula of the variant mass for an accelerated charged particle

\section{Power energy emitted for an Accelerated Charged Particle}

The kinetic formula for an accelerated charged particle is as follows:

$K=m_{o} c^{2}-m c^{2}$

$K=m_{o} c^{2}\left(1-\frac{1}{\sqrt{\left(1+\frac{v^{2}}{c^{2}}\right)}}\right)$

$\frac{d K}{d t}=-c^{2} \frac{d m}{d t}$ (the change in the kinetic energy of the particle causes a proportional change in its mass, the minus sign is due to the mass decreases)

$$
K=m_{o} c^{2}\left(1-\left(1+\frac{v^{2}}{c^{2}}\right)^{-1 / 2}\right)
$$

By doing the respective derivation, it is obtained:

$\mathrm{P}=\mathrm{dk} / \mathrm{dt}$

$\frac{d K}{d t}=\frac{m_{o} v}{\left(1+\frac{v^{2}}{c^{2}}\right)^{3 / 2}} \frac{d v}{d t}$ 
$P=\frac{m_{o} v}{\left(1+\frac{v^{2}}{c^{2}}\right)^{3 / 2}} a$ (formula of the power energy for an accelerated charged particle).

The term in parenthesis can be expressed as follows:

$\left(1+\frac{v^{2}}{c^{2}}\right)^{-\frac{3}{2}}=1-\frac{3}{2} \frac{v^{2}}{c^{2}}+\frac{15}{8} \frac{v^{4}}{c^{4}}-\cdots$

For $\mathrm{v}<<\mathrm{c}$, it is achieved:

$P=m_{o} v a\left(1-\frac{3}{2} \frac{v^{2}}{c^{2}}\right)$ for very low velocities

If this formula is compared with the Larmor formula (which is used to calculate the power radiated by a non-relativistic point accelerated charge, electromagnetic theory [7], [8], [9], [10]):

$P=\frac{q^{2} a^{2}}{6 \pi \varepsilon_{0} c^{3}}$ or in CGS units: $P=\frac{2 q^{2} a^{2}}{3 c^{3}}$

$\frac{q^{2} a^{2}}{6 \pi \varepsilon_{o} c^{3}}=m_{o} v\left(1-\frac{3}{2} \frac{v^{2}}{c^{2}}\right) a$

$a=\frac{6 \pi \varepsilon_{o} c^{3}}{q^{2}} m_{o} v\left(1-\frac{3}{2} \frac{v^{2}}{c^{2}}\right)$ where $\mathrm{q}$ is the charge of the particle.

Or if it is used the equivalent formula:

$P=\frac{2}{3} \frac{m_{o} r_{e} a^{2}}{c}$, it is obtained:

$\frac{2}{3} \frac{m_{o} r_{e} a^{2}}{c}=m_{o} v\left(1-\frac{3}{2} \frac{v^{2}}{c^{2}}\right) a$

$a=\frac{3 v c}{2 r_{e}}\left(1-\frac{3}{2} \frac{v^{2}}{c^{2}}\right)$ where $\mathrm{r}_{\mathrm{e}}$ is the classic electron radius: $\mathrm{r}_{\mathrm{e}}=2,82 * 10^{-15} \mathrm{~m}$

\section{Conclusions}

The kinetic formula represents the emission of the electromagnetic radiation for an accelerated charged particle. It is:

$K=m_{o} c^{2}\left(1-\frac{1}{\sqrt{\left(1+\frac{v^{2}}{c^{2}}\right)}}\right)$

$\mathrm{K}=\mathrm{m}_{\mathrm{o}} \mathrm{c}^{2}-\mathrm{mc} \mathrm{c}^{2}$

$\mathrm{K}=\mathrm{hf}$ (it corresponds to the ionization energy at the atom of Hydrogen or the energy of the photon emitted or the electromagnetic radiation emitted by the accelerated charged particle).

For higher velocities (when $\mathrm{v}$ is approaching to $\infty$ ), the kinetic energy has a maximum value of $\mathrm{m}_{0} \mathrm{c}^{2}$, which correspond to the rest mass of the particle. It is completely emitted as electromagnetic radiation at higher velocities. The mass of the particle after the emission of the electromagnetic radiation (losing the respective mass-energy of the electromagnetic radiation and equal to the lost mass of the particle) is: 
$m=\frac{m_{0}}{\sqrt{1+\frac{v^{2}}{c^{2}}}}$ (mass of the particle after the emission of the electromagnetic radiation)

$m=\frac{m_{o} c^{2}}{\sqrt{1+\frac{v^{2}}{c^{2}}}} / c^{2}$ (mass of the particle in units of energy: $\mathrm{eV} / \mathrm{c}^{2}$ )

Also, it is equals to:

$\mathrm{mc}^{2}=\mathrm{m}_{\mathrm{o}} \mathrm{c}^{2}-(\mathrm{hf})$ where $\mathrm{hf}$ is the energy of the photon emitted or emission of the energy of the electromagnetic radiation.

At the mass formula, when the velocity $\mathrm{v}$ is increasing, the mass $\mathrm{m}$ is decreasing too until $\mathrm{v}$ is very high $(\infty)$ and the value of mass is very low (approximately to 0 ). At this point, almost all the mass is emitted as electromagnetic energy. Thus, the velocity v can get values higher than the light velocity because of the decreasing mass. The lost mass is equivalent to the mass-energy of the electromagnetic radiation.

These formulas have been verified by means of theory, thought experiments, experiments and in the hydrogen atom.

The formula of the power energy for an accelerated charged particle is as follows:

$P=\frac{m_{o} v}{\left(1+\frac{v^{2}}{c^{2}}\right)^{3 / 2}} a$

For very low velocities, it is as follows:

$P=m_{o} v a\left(1-\frac{3}{2} \frac{v^{2}}{c^{2}}\right)$

The acceleration for very low velocities is as follows:

$a=\frac{6 \pi \varepsilon_{o} c^{3}}{q^{2}} m_{o} v\left(1-\frac{3}{2} \frac{v^{2}}{c^{2}}\right)$ where $\mathrm{q}$ is the charge of the particle

or:

$a=\frac{3 v c}{2 r_{e}}\left(1-\frac{3}{2} \frac{v^{2}}{c^{2}}\right)$ where $\mathrm{r}_{\mathrm{e}}$ is the classic electron radius.

\section{References}

[1] Einstein, Lorentz, Weyl and Minkowski, The Principle of Relativity, Publicaciones Dover, 1923, p. 29.

[2] John P. McKelvey Howard Grotch, Física para ciencias e ingeniería 2, Editorial Harla, México, 1981.

[3] Daniele Sasso, Dynamics and electrodynamics of moving real systems in the Theory of Reference Frames, January 2010.

[4] Robert Resnick, Introducción a la Teoría Especial de la Relatividad, Editorial Limusa, México, 1981.

[5] Leif Holmlid and Sveinn Olafsson, Detection of muons and neutral kaons from ultra- dense hydrogen H(o) by lepton pair-production, University of Gothenburg, November 2019. 
Asian Journal of Basic Science \& Research Volume 2, Issue 3, Pages 43-59, July-September 2020

[6] Guillermo Alegría de la Colina, Física Moderna 2, Física Atómica, Editorial Limusa, México, 1981.

[7] William Hayt, JR., Teoría Electromagnética, Editorial McGraw-Hill, México, 1991.

[8] Joseph A. Edminister, Electromagnetismo, Editorial McGraw-Hill, Serie de Compendios Schaum, México, 1992.

[9] Carl T.A. Johnk, Teoría Electromagnética, Principios y Aplicaciones, Editorial Limusa, México, 1984.

[10] Ashok K. Singal, Compatibility of Larmor's Formula with Radiation Reaction for an Accelerated Charge, 2015. 